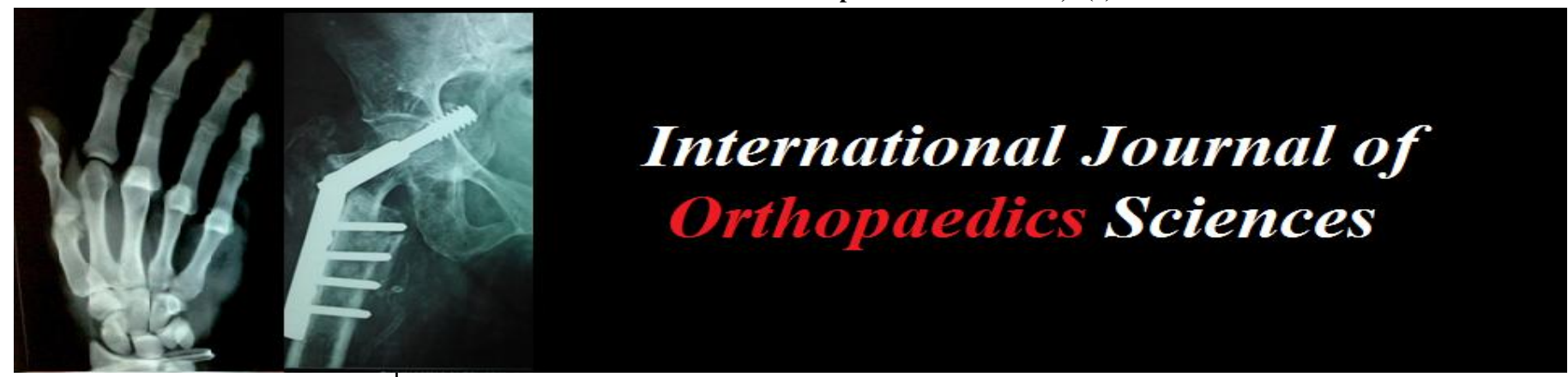

ISSN: $2395-1958$

IJOS 2017; 3(3): 901-907

(C) 2017 IJOS

www.orthopaper.com

Received: 03-05-2017

Accepted: 02-06-2017

Dr. M. Seran

M Assistant Professor, Department Of Orthopedics, Government Stanley Medical College, Tamil Nadu, India

Prof. Dr. S. Veera Kumar

S Professor, Department Of

Orthopedics, Government

Stanley Medical College, Tamil

Nadu, India

Dr. S. Kathir Azhagan

S Resident Department of

Orthopedics, Government

Stanley Medical College, Tamil

Nadu, India

Correspondence

Prof. Dr. S. Veera Kumar

S Professor, Department Of

Orthopedics, Government

Stanley Medical College, Tamil

Nadu, India

\section{Posterior plating of tibial shaft fractures: A functional outcome analysis}

\author{
Dr. M. Seran, Prof. Dr. S. Veera Kumar and Dr. S. Kathir Azhagan
}

DOI: https://doi.org/10.22271/ortho.2017.v3.i3m.132

\section{Abstract}

Tibial shaft fractures are the most common of long bone fractures. Tibial fractures are usually the result of trauma, both high energy and low energy. Increasing energy of injury leads on to both bone and soft tissue complications. Approximately $25 \%$ of both bone fractures of leg are open fractures requiring external fixation or wound debridement with resultant problems in wound healing by secondary intention. A few often require secondary procedures for internal fixation which might get infected leading on to a vicious cycle implant exit, external fixation, wound debridement. Posterior tibial plating aims at avoiding the vicious cycle by providing primary internal fixation. We have reported a case series of patients who got operated for fracture tibia with posterior tibial plating, patients were followed up and functional outcome is reported in this study.

Materials \& Methods: Patients who had sustained lower limb fractures were examined and those patients with fracture at distal diametaphyseal region of tibia along with minor soft tissue injuries (Gustilo Anderson Type I and Type II) were chosen. Patients with fracture both bone leg with open physes were also included. We treated three patients with posterior plating of tibia.

Results: Bone Union was achieved in all three patients with all of them bearing full weight at the end of four months.

Conclusion: Posterior tibial plating adds another viable alternative in treating distal diametaphyseal fractures with soft tissue compromise or open physes.

Keywords: posterior plating, tibial fractures

\section{Introduction}

Tibial fractures caused by high-energy trauma are often associated with severe soft tissue in jury. While the general consensus among surgeons is to operate and internally fix these types of fracture; the choice of implants and approach is variable. Fractures with comminution associated with predominant large butterfly split component in the posterior coronal plane are difficult to reduce and achieve fixation by conventional means

\section{Current treatment options}

There are four different methods for treating tibial shaft fractures and the goal of each method is to stabilize the bone in an acceptable alignment.

1. Long leg casting/ patellar tendon bearing cast/ functional bracing

2. Plate fixation - locked /non-locked, open /minimally invasive

3. Intramedullary nailing - reamed/unreamed, static/dynamic locking/elastic nailing

4. External fixators- uniplanar/multiplanar fixators

Intramedullary nailing is advocated as the treatment for diaphyseal fractures. Distal diametaphyseal fractures often presents some technical difficulties due to the anatomy of distal diametaphyseal flare, the proximity of the fracture to the joint line and there is also a known association of posterior malleolar, medial malleolar and lateral malleolar fractures.

\section{Posterior plating through posterolateral approach}

Traditionally a posterolateral approach to the tibia has been used for bone grafting when the soft tissues overlying anterior aspect of the tibia are not of good quality, due to the presence of open wounds or scars. 
Posterior plating of tibia through posterolateral approach offers a new dimension in treating diametaphyseal fractures of tibia with poor skin condition over anterior, anteromedial aspect of leg and also in treating tibial fractures with open epiphysis. Other benefits of this approach include dual plating both tibia and fibula, access to the posterior Malleolus and medial malleolus, improved bone healing and wound healing, adequate soft tissue coverage and of course less hardware prominence

\section{Approach}

1. Position the patient prone or on the side, with the affected extremity uppermost.

2. Make the skin incision the desired length along the lateral border of the gastrocnemius muscle on the posterolateral aspect of the leg.

3. Develop the plane between the gastrocnemius, the soleus, and the flexor hallucis longus muscles posteriorly and the peroneal muscles anteriorly. Find the lateral border of the soleus muscle and retract it and the gastrocnemius muscle medially and posteriorly; arising from the posterior surface of the fibula is the flexor hallucis longus

4. Detach the distal part of the origin of the soleus muscle from the fibula, and retract it posteriorly and medially

5. Continue the dissection medially across the interosseous membrane, detaching those fibers of the posterior tibial muscle arising from it

6. Follow the interosseous membrane to the lateral border of the tibia and detach subperiosteally the muscles that arise from the posterior surface of the tibia

7. The posterior half of the fibula lies in the lateral part of the wound; its entire shaft can be explored. The flat posterior surface of the tibial shaft can be completely exposed except for its proximal fourth.

\section{Case summary 1}

Rajeswari, female patient aged 40 had sustained an Road traffic accident on examination she had a grade I open fracture of both bone right leg with a $1 \mathrm{~cm}$ laceration above medial malleolus and deep abrasions over the antero medial aspect of right leg. Primary skin suturing was done after thorough wound wash. She was managed with intravenous antibiotics and daily wound dressing.

She was taken up for posterior plating of tibia \& fibular plating through posterolateral approach.
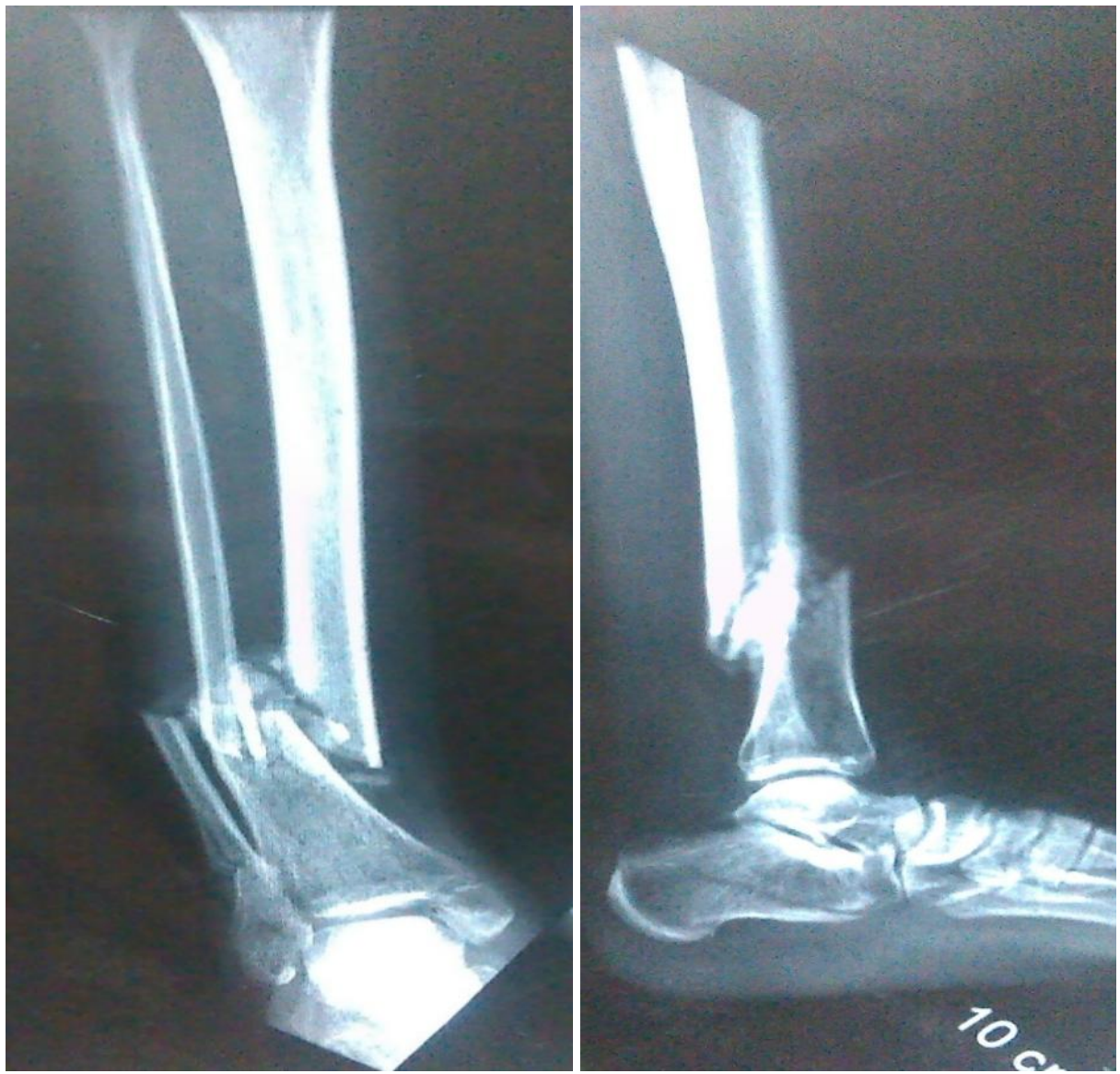

Pre-Operative Radiograph 


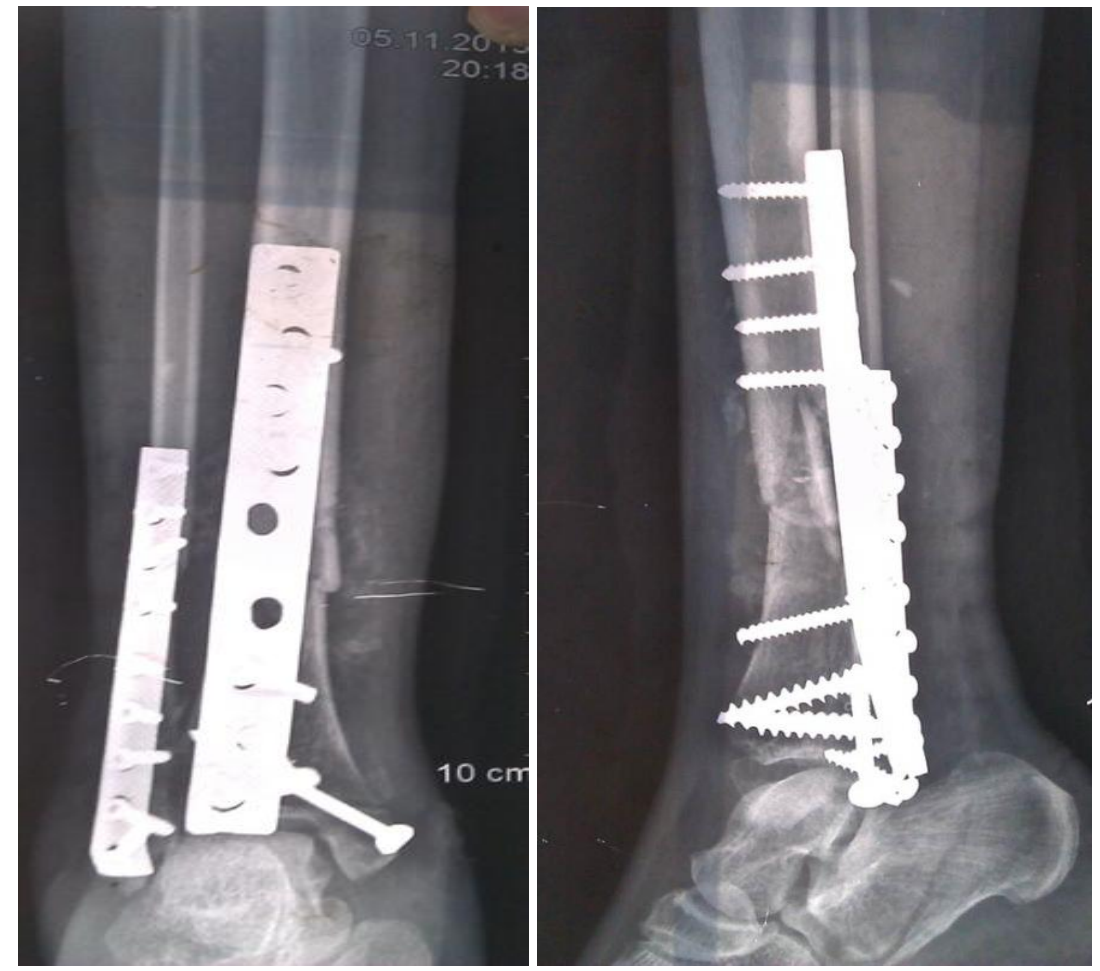

Post-Operative Radiograph

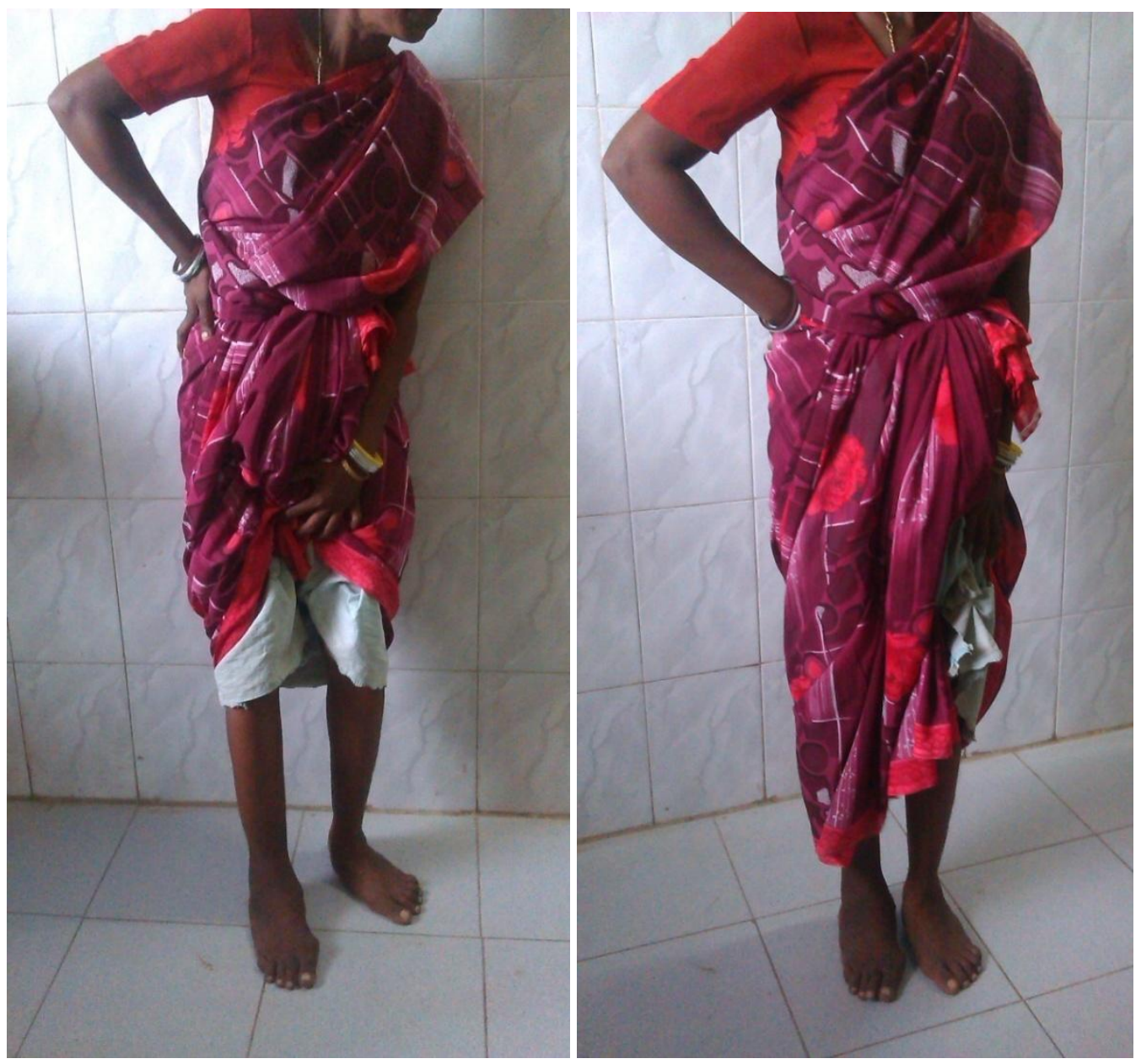

Weight bearing clinical picture after 4 months 


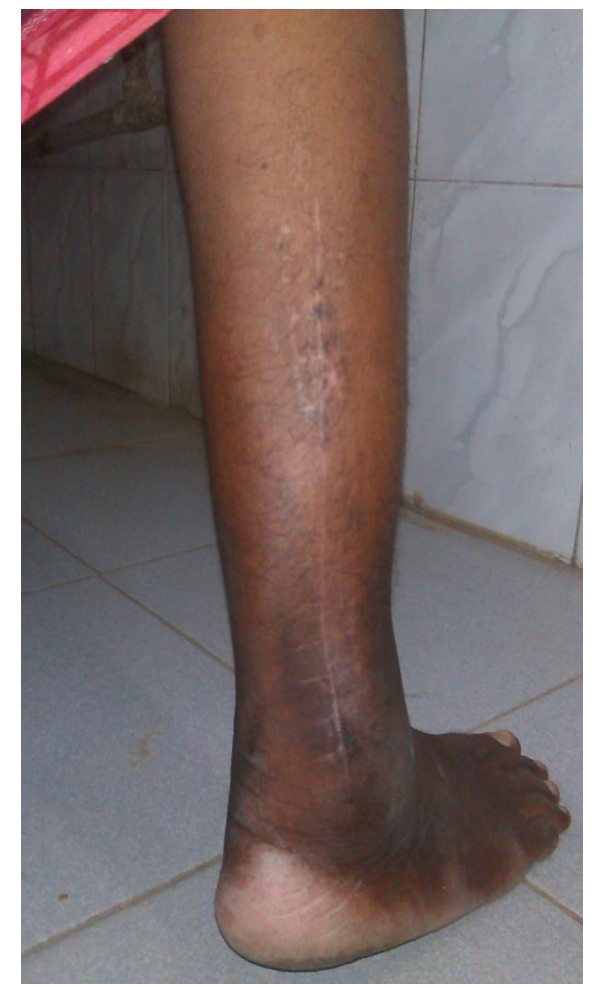

Healed Surgical Scar

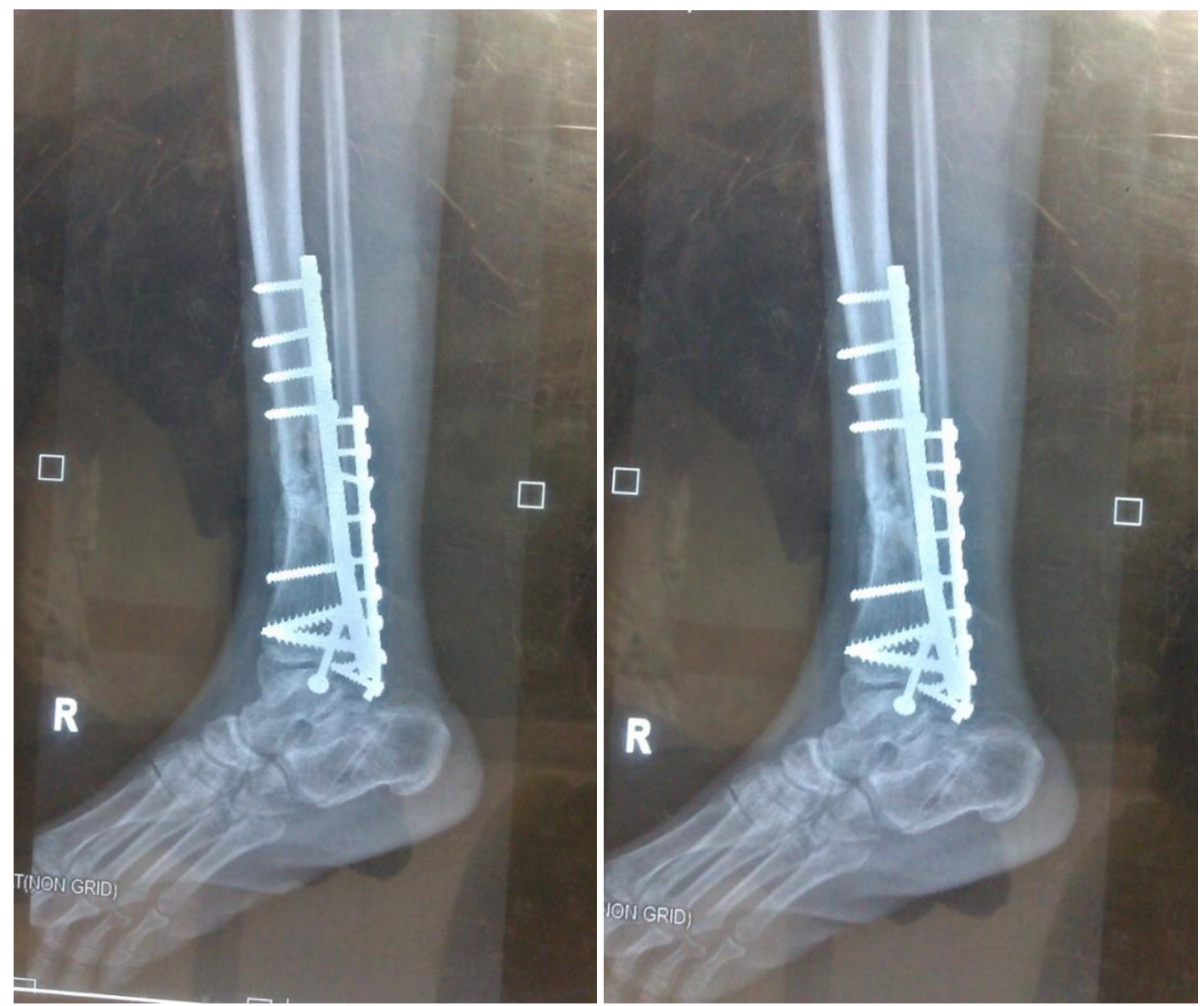

Follow Up Radiograph at 4 Months Showing Consolidation

Case Summary II

Suresh aged 40 had sustained an RTA and was admitted with grade one open both bone fracture distal one third. He was managed by posterior plating 

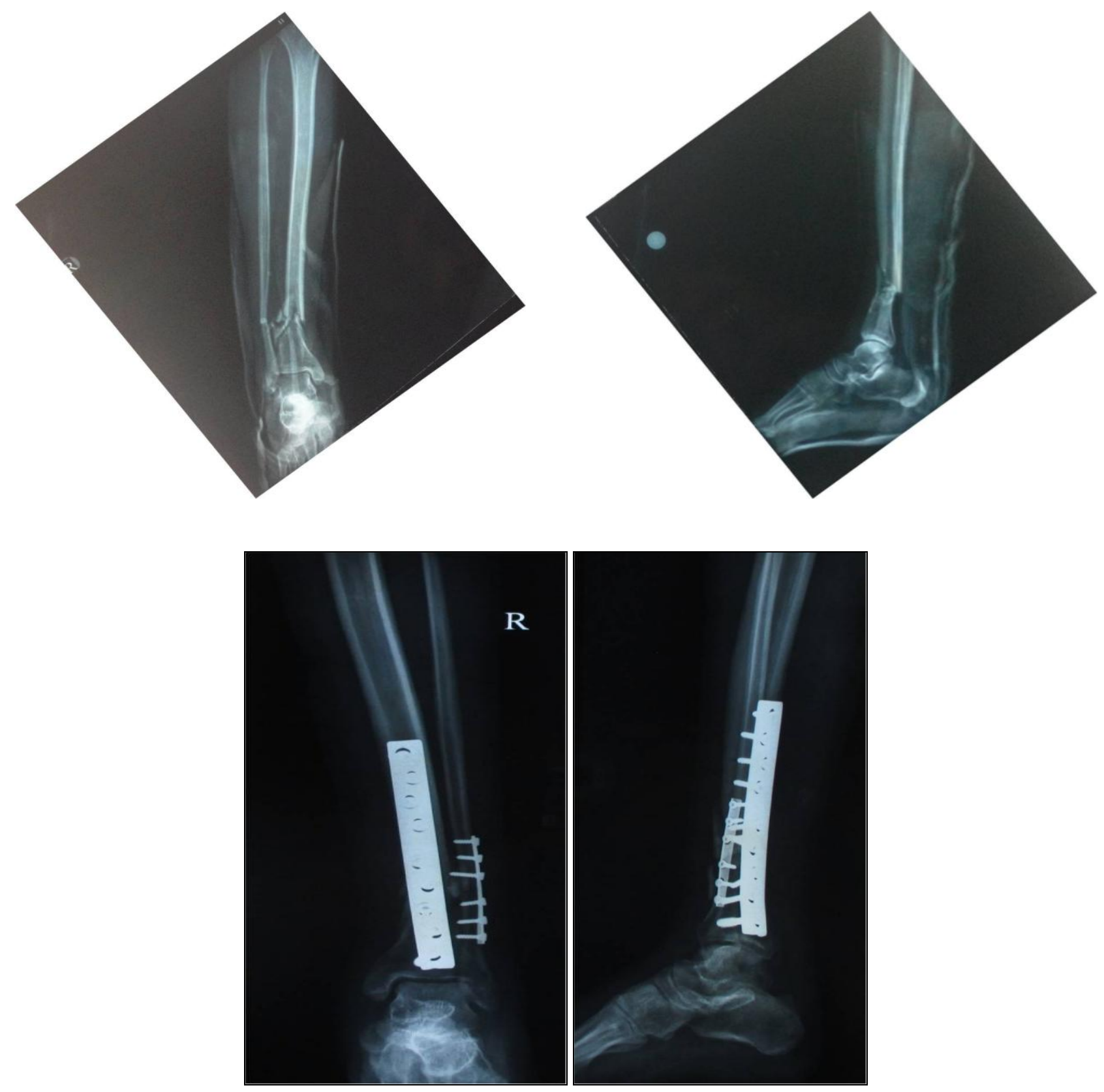

\section{Case Summary III}

Prabhu aged 14 had sustained an RTA and suffered both bone fracture of mid one third leg. He was managed by posterior plating
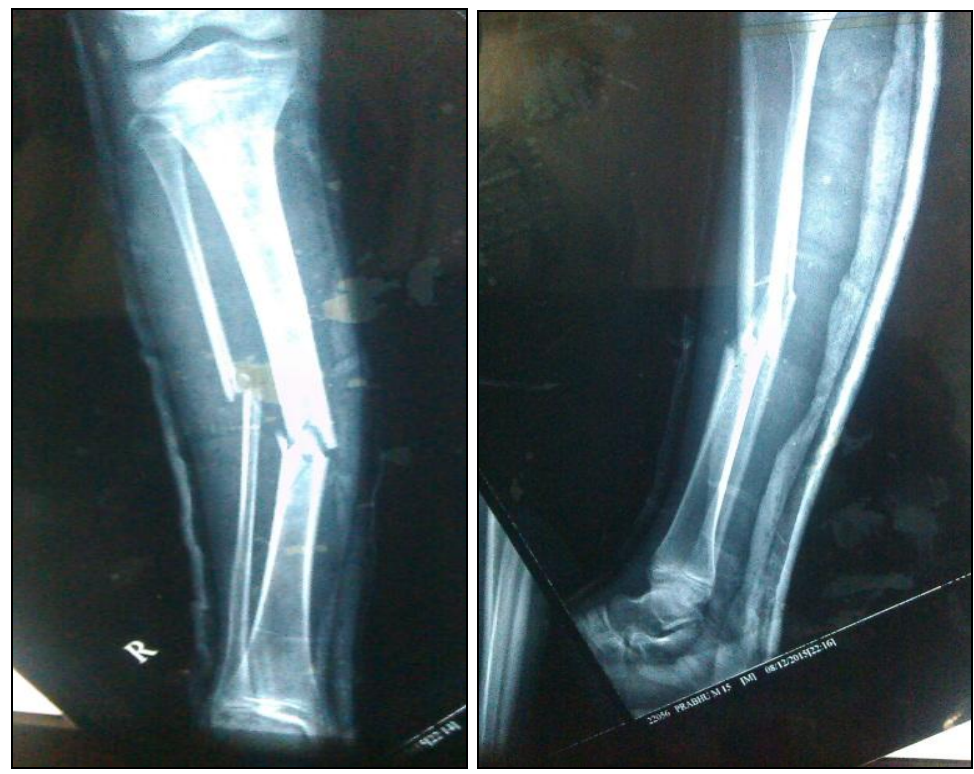

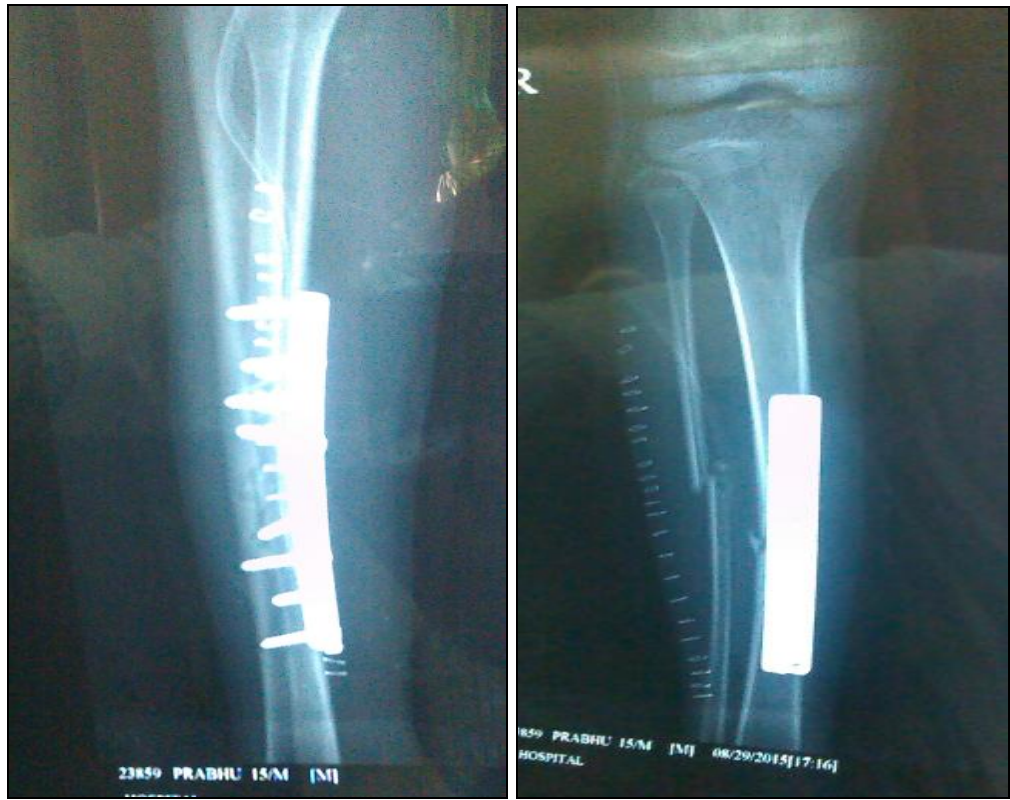

Follow up radiograph at 4 months showing consolidation
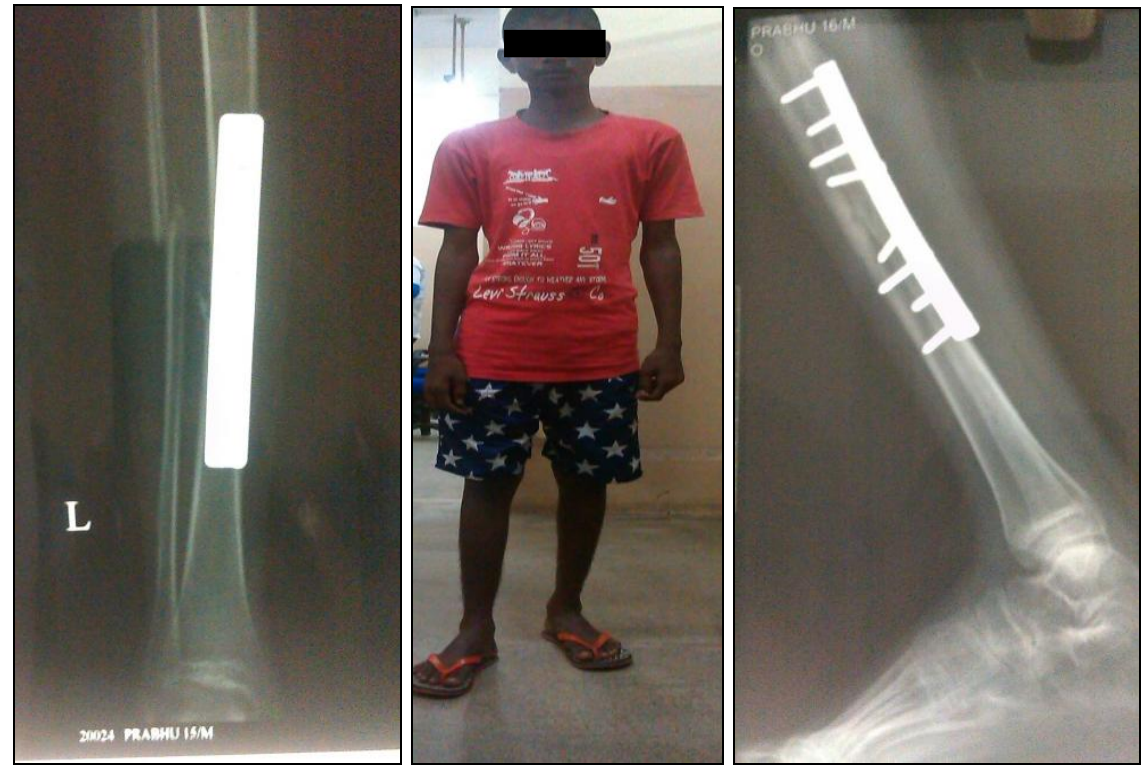

\section{Results}

No cases of infection were observed. All Three Patients showed bony consolidation and full weight bearing at an average of 4 months. No loss of reduction were observed. There were no growth disturbance found in patients with open physis.

\section{Discussion}

Tibial fractures are the most common long bone fracture in the body. Current surgical treatment options for tibial shaft fractures are nailing, plating, simple external fixator and circular external fixator. Most Diaphyseal fractures are treated by nailing or plating, however treatment options are made considering soft tissue status fracture type and age of the patient.

The amount of malalignment and shortening considered acceptable also is controversial. Tarr et al. and Puno et al. demonstrated that distal tibial malalignment may be more poorly tolerated than more proximal malalignment. The recommendations in the literature vary widely: 4 to 10 degrees of varus-valgus malalignment, 5 to 20 degrees of anteroposterior malalignment, 5 to 20 degrees of rotatory malalignment, and 10 to $20 \mathrm{~mm}$ of shortening. In general, we agree with Trafton's recommendation and strive to achieve less than5 degrees of varus-valgus angulation, less than 10 degrees of anteroposterior angulation, less than 10 degrees of rotation, and less than $15 \mathrm{~mm}$ of shortening. Maintaining fracturealignment is difficult in certain fracture types, and if repeatedattempts at realignment have been unsuccessful, operativefixation is indicated.

The important factors in prognosis are (1) the amount of initial displacement, (2) the degree of comminution, (3) whether infection has developed, and (4) the severity of thesoft tissue injury excluding infection

Distal diametaphyseal fractures when associated with skin and soft tissue compromise on the anterior and medial surface of leg leads to difficulty in distal locking of intramedullary nail. Indications for Posterior plating are (1) a tibial shaft fracture with periarticular metaphyseal comminution that precludes locked intramedullary nailing, (2) softtissue damage of such severity that it prohibits the use of standard incisions,(3)patients with open physes, anatomical deformity, burns or wounds over the entry portal, or mosttype IIIC open fractures.

Posterior tibia plating offers a safe corridor for distal fractures of tibia where antero medial soft tissue compromise has 
precluded standard nailing or plating and in fractures with open physes. Other benefits of this approach include dual plating both tibia and fibula, access to the posterior malleolus, improved bone healing and wound healing, adequate soft tissue coverage and of course less hardware prominence.

We, in our series had three patients with conditions precluding standard nailing or conventional plating and were treated by posterior plating through posterolateral approach.

\section{Conclusion}

Posterior plating of tibia through posterolateral approach is a viable alternative for treating distal diaphyseal-metaphyseal fractures of tibia and fibula. There were no wound infections or wound breakdown and implant prominence and other implant related complications were nil due to adequate soft tissue cover. In our series all three patients showed fracture consolidation at 4 months post fixation and all patients could able to bear weight without any abnormal consequences.

\section{References}

1. Fractures of lower extremity. In: Canale ST, ed. Campbell's operative ortho paedics, $\mathrm{M}$.

2. Fracture of Adults -Rockwood and Green -Eighth edition

3. Kyle RF. Biomechanics of intramedullary fracture fixation J Orthopedics. 1985; 8(11):1356-9.

4. Montu Jain, Roop Singh. Arun Madharia-Posterior coronal plating for tibial fractures: technique and advantages: Chinese Journal of Traumatology. 2014; 17(2):84-87. 